\title{
Periventricular anastomosis in moyamoya disease: detecting fragile collateral vessels with MR angiography
}

\author{
Takeshi Funaki, MD, PhD, ${ }^{1}$ Jun C. Takahashi, MD, PhD, ${ }^{2}$ Kazumichi Yoshida, MD, PhD, ${ }^{1}$ \\ Yasushi Takagi, MD, PhD, ${ }^{1}$ Yasutaka Fushimi, MD, PhD, ${ }^{3}$ Takayuki Kikuchi, MD, PhD, ${ }^{1}$ \\ Yohei Mineharu, MD, PhD, ${ }^{1}$ Tomohisa Okada, MD, PhD, ${ }^{3}$ Takaaki Morimoto, MD, ${ }^{1}$ and \\ Susumu Miyamoto, MD, $\mathrm{PhD}^{1}$
}

Departments of ${ }^{1}$ Neurosurgery and ${ }^{3}$ Diagnostic Imaging and Nuclear Medicine, Kyoto University Graduate School of Medicine, Kyoto; and 'Department of Neurosurgery, National Cerebral and Cardiovascular Center, Suita, Osaka, Japan

OBJECTIVE The authors' aim in this paper was to determine whether periventricular anastomosis, a novel term for the abnormal collateral vessels typical of moyamoya disease, is reliably measured with MR angiography and is associated with intracranial hemorrhage.

METHODS This cross-sectional study sampled consecutive patients with moyamoya disease or moyamoya syndrome at a single institution. Periventricular anastomoses were detected using MR angiography images reformatted as slidingthin-slab maximum-intensity-projection coronal images and were scored according to 3 subtypes: lenticulostriate, thalamic, and choroidal types. The association between periventricular anastomosis and hemorrhagic presentation at onset was evaluated using multivariate analyses.

RESULTS Of 136 eligible patients, 122 were analyzed. Eighteen (14.8\%) patients presented with intracranial hemorrhage with neurological symptoms at onset. Intra- and interrater agreement for rating of the periventricular anastomosis score was good ( $\kappa_{w}=0.65$ and 0.70 , respectively). The prevalence of hemorrhagic presentation increased with the periventricular anastomosis score: $2.8 \%$ for Score $0,8.8 \%$ for Score 1, $18.9 \%$ for Score 2, and $46.7 \%$ for Score 3 ( $p<0.01$ for trend). Univariate analysis revealed that age $(p=0.02)$ and periventricular anastomosis score $(p<0.01)$ were factors tentatively associated with hemorrhagic presentation. The score remained statistically significant after adjustment for age (OR 3.38 [95\% Cl 1.84-7.00]).

CONCLUSIONS The results suggest that periventricular anastomosis detected with MR angiography can be scored with good intra- and interrater reliability and is associated with hemorrhagic presentation at onset in moyamoya disease. The clinical utility of periventricular anastomosis as a predictor for hemorrhage should be validated in further prospective studies.

http://thejns.org/doi/abs/10.3171/2015.6.JNS15845

KEY WORDS moyamoya disease; cerebral hemorrhage; magnetic resonance angiography; periventricular anastomosis; sensitivity and specificity; reproducibility; vascular disorders

$\mathrm{M}$ OYAMOYa disease is characterized by chronic progressive stenosis of the terminal portion of the bilateral internal carotid artery. Development of numerous abnormal collateral vessels at the base of the brain, another characteristic of moyamoya disease, might be of clinical importance in relation to hemorrhage. ${ }^{15}$ Periventricular anastomosis is a novel term for such collaterals according to vascular morphology ${ }^{5}$ and is defined as anastomosis between the perforating or choroidal artery and medullary artery in the periventricular area. Although several researchers have documented some characteristics of such anastomoses, ${ }^{2,11,14,22}$ we are among the first to have taken a systemic approach to the task. In moyamoya disease, cerebral microbleeds are most commonly observed in the periventricular area. ${ }^{11,18,20}$ It thus seems reasonable to hypothesize that periventricular anastomosis is related to hemorrhage. This hypothesis has, however, remained unproven. 
Sliding-thin-slab maximum-intensity projection (STSMIP) is a common imaging reformation for CT or MR angiography (MRA) that facilitates visualization of target vessels by eliminating the effect of other vessels overlapping and obscuring the view.,12 Time-of-flight MRA reformatted using STS-MIP might be useful in assessing periventricular anastomosis. No previous study, however, has documented such usefulness, and information on the reliability of the imaging modality in terms of detecting anastomosis is lacking.

This cross-sectional study had 2 objectives: to estimate the reliability of the detection and scoring of periventricular anastomosis with MRA and to determine whether periventricular anastomosis detected with MRA is associated with hemorrhage in moyamoya disease.

\section{Methods}

This study was approved by the ethics committee of the Kyoto University Graduate School of Medicine.

\section{Participants and Setting}

All patients with moyamoya disease or moyamoya syndrome who first visited Kyoto University Hospital after 2009 were eligible for the study. Diagnosis of moyamoya disease was determined according to the proposed criteria. 19 "Moyamoya syndrome" is defined as a secondary moyamoya phenomenon caused by accompanying disorders such as autoimmune disease, meningitis, brain tumor, hyperthyroidism, Down syndrome, neurofibromatosis Type 1, or a history of head irradiation. Patients who had undergone MRI and did not meet the imaging requirements indicated below and who had already undergone bypass surgery or another specific treatment elsewhere were excluded from the study. The study population was consecutively sampled. All data for the study were collected during the first visit or upon admission.

\section{Imaging Acquisition and Processing}

A 3-T MR scanner (Magnetom Trio, Siemens), which successfully reveals abnormal collateral vessels in moyamoya disease, ${ }^{6}$ was introduced in 2009 for image acquisition in patients with moyamoya disease. The time-of-flight MRA source images were scanned as usual axial sections with a 32-channel head coil under the following parameters: $20-\mathrm{msec}$ repetition time, $3.7-\mathrm{msec}$ echo time, $20^{\circ}$ flip angle, $0.7-\mathrm{mm}$ slice thickness, $220 \times 172-\mathrm{mm}$ field of view with a $384 \times 300$ matrix, and $0.57 \times 0.57-\mathrm{mm}$ pixel size. The imaging field extended from the level of the foramen magnum to beyond the upper margin of the body of the lateral ventricle. The generalized autocalibrating partially parallel acquisition (GRAPPA) technique ${ }^{7}$ was used to acquire a relatively wide scan field in less scan time (5 minutes and 34 seconds).

The MRA data thus acquired were reformatted as STSMIP images in a coronal plane perpendicular to the lateral ventricle and represented as 5- to 15-mm-thick slabs of overlapping volumes (Fig. 1). A window width of 250 and a window level of 120 arbitrary units were used to differentiate the vessels, ventricle, and parenchyma. STS-MIP images were generated on a workstation integrated into the picture-archiving and communication system (Aquarius iNtuition Viewer version 4.4.7, TeraRecon, Inc.).

\section{Measurement of Periventricular Anastomosis}

Periventricular anastomosis, a primary factor of interest, was measured using STS-MIP MRA. Presence of anastomosis was determined by a rater (T.F.) who was blinded to other clinical information. To estimate intrarater reliability, the rater rated the images twice at a 1-month interval on a subsample of the last 34 consecutive patients. Another rater (T.M.) independently rated images from the same subsample to determine interrater reliability. Raters had participated in an advance self-training session on 10 representative cases in which periventricular anastomosis had been confirmed.

The classification and definition of periventricular anastomosis followed that of our previous study. ${ }^{5}$ In brief, anastomosis was defined as a connection beginning at the perforating or choroidal arteries and ending at the medial end of the medullary or insular arteries in the periventricular area. Anastomoses were classified into 3 subtypes (Fig. 1): 1) lenticulostriate, beginning at the lenticulostriate artery and connecting to the medullary artery at the lateral corner of the frontal horn or body of the lateral ventricle; 2) thalamic, beginning at the thalamotuberal or thalamoperforating arteries and connecting to the medullary or insular arteries beneath the ependyma of the lateral or third ventricle (medial type), or beginning at the thalamogeniculate (or rarely, choroidal) artery and connecting to the insular artery superior to the inferior horn or at the lateral corner of the body of the lateral ventricle (lateral type); and 3) choroidal, beginning at the plexal segment of the anterior or posterior choroidal arteries and connecting to the medullary artery beneath the lateral wall of the trigon of the lateral ventricle. Detection of at least the proximal part of the medullary artery was considered a positive indicator for the presence of periventricular anastomosis. For the thalamic type, a vessel signal extending outside the thalamus or inside the third ventricle was judged as a positive indicator. Each subtype of anastomosis was determined as "present" with a positive finding in at least one hemisphere, and determined as "absent" with a negative finding in both hemispheres. Each subtype was scored 1 for "present" and 0 for "absent," and the sum of these subscale scores for each patient represented the periventricular anastomosis score; the scores thus ranged from 0 to 3 .

To avoid information bias from detection of hemorrhage, MRA images from the chronic phase were used for the assessment of patients who had experienced hemorrhagic stroke.

\section{Other Variables}

The outcome variable was hemorrhage as a mode of presentation (hemorrhagic presentation), defined as intracranial hemorrhage at onset causing any neurological symptom and detected with CT.

Possible confounders affecting results, including age, sex, concurrence of disorders causing moyamoya syndrome, history of hypertension and diabetes, unilateral 

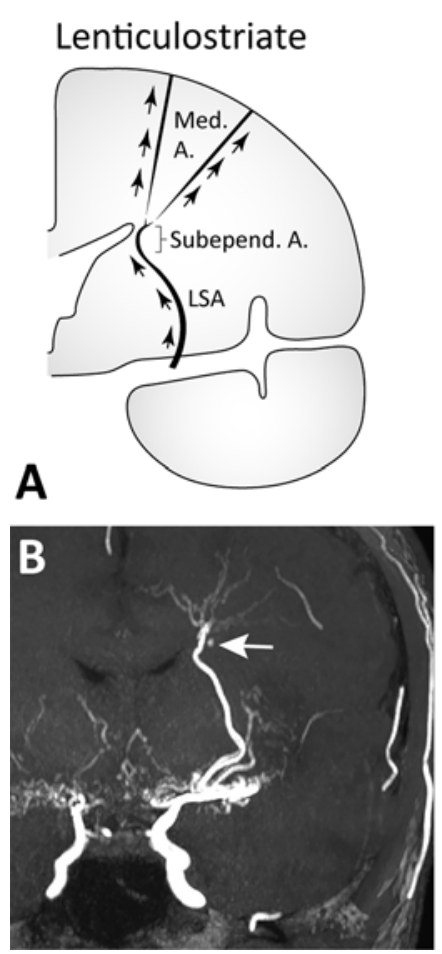

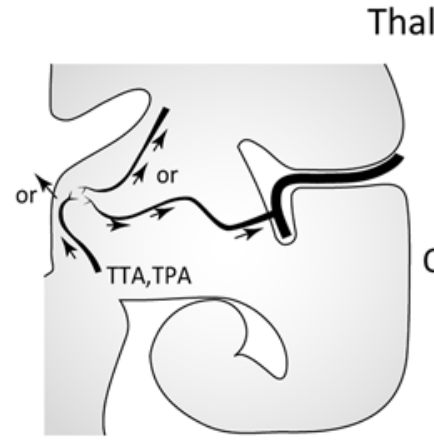

(medial)

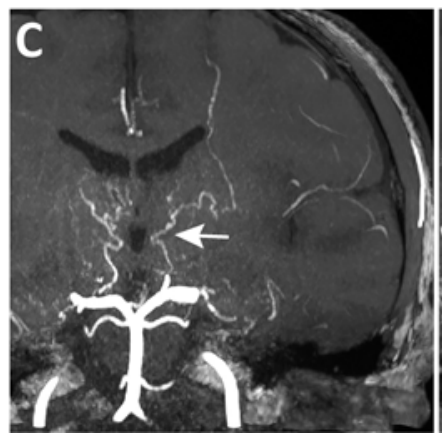

Thalamic

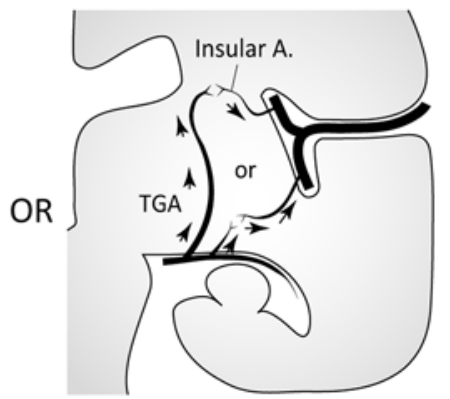

(lateral)

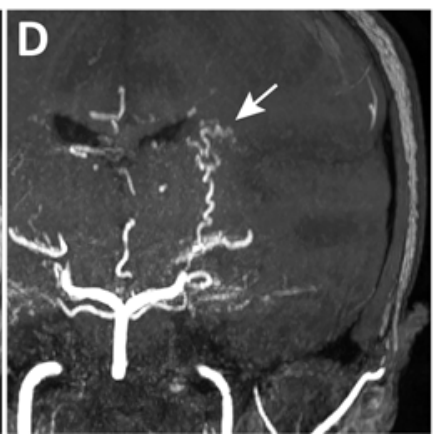

\section{Choroidal}
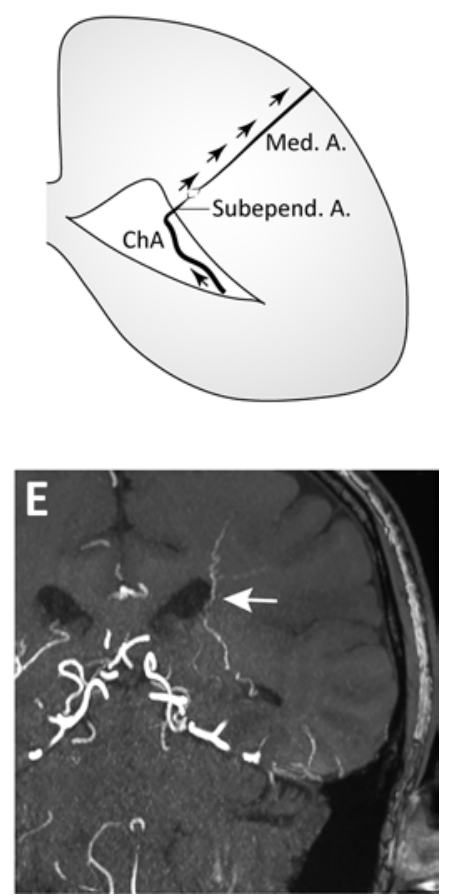

FIG. 1. Schematic illustration (A) and STS-MIP MR angiograms (B-E) showing periventricular anastomosis. B: Lenticulostriate type. C and D: Thalamic type. E: Choroidal type. The periventricular anastomosis score is 3 (lenticulostriate, 1; thalamic, 1; and choroidal, 1) given all images are from the same patient. $A=$ artery; $C h A=$ choroidal artery; LSA = lenticulostriate artery; Med = medullary; subepend $=$ subependymal; TGA = thalamogeniculate artery; TPA = thalamoperforating artery; TTA = thalamotuberal artery. Panel A: Modified with permission from Funaki et al: Neurol Med Chir (Tokyo) 55:204-209, 2015.

disease, and evidence of severe hemodynamic compromise, were also measured. Hypertension is diagnosed if systolic and diastolic blood pressure at first visit or on admission exceeded $140 \mathrm{~mm} \mathrm{Hg}$ and $90 \mathrm{~mm} \mathrm{Hg}$, respectively, or if the patient was receiving antihypertensive agents. Diabetes mellitus was defined as use of insulin or oral glucose inhibitors. Severe hemodynamic compromise was defined as a defect in the cerebral blood flow both at rest and after acetazolamide challenge revealed in single photon emission computed tomography.

\section{Statistical Analysis}

Intra- and interrater agreement on the presence of each periventricular anastomosis was assessed using the kappa statistic ( $\kappa)$, and agreement on rating the periventricular anastomosis score was assessed using weighted kappa $\left(\kappa_{\mathrm{w}}\right)$. The Wilcoxon rank-sum test and Fisher's exact test were used as appropriate for the comparison of baseline characteristics. The Cochrane and Armitage test for trend ${ }^{1}$ was used to assess the trend in an ordinal variable. Significant factors in the univariate baseline comparison were incorporated into multiple logistic regression models. The periventricular anastomosis score was analyzed not only as an ordinal variable but also as an interval variable and was incorporated into models. We considered $\mathrm{p}$ values of $<0.05$ and $95 \%$ CI of OR not including 1 significant. All analyses were performed using JMP software (version 11, SAS Institute Inc.).

\section{Results}

A total of 136 patients were diagnosed with moyamoya disease or moyamoya syndrome at our hospital between January 2009 and August 2014. Fourteen patients were excluded from the study for the following reasons: the MRA scan field was not sufficiently wide (6 patients); the assessment of MRI was difficult because of metallic artifact (1 patient); the patient had already undergone bypass surgery at another hospital (6 patients); and the patient had undergone coil embolization due to a peripheral aneurysm in the anterior choroidal artery (1 patient).

Of the remaining 122 patients included in the analysis, $18(14.8 \%)$ had presented with hemorrhage at onset, 66 (54.1\%) with ischemic attack, 19 (15.6\%) with cerebral infarction, and $3(2.5 \%)$ with seizure. Sixteen patients (13.1\%) were asymptomatic or had subtle symptoms such as headache.

\section{Reliability}

Intrarater and interrater reliability for the presence of each subtype of periventricular anastomosis are summarized in Table 1 . Very good agreement was observed for the lenticulostriate type $(\kappa=0.85$ for intrarater agreement and 0.93 for interrater agreement). Agreement for the choroidal type ranged from moderate to good $(\kappa=0.70$ for intrarater agreement and 0.54 for interrater agreement). Agreement for the thalamic types was moderate $(\kappa=$ 
TABLE 1. Cross tabulation, sensitivity, specificity, and intra- and interrater agreement for each type of periventricular anastomosis

\begin{tabular}{|c|c|c|c|c|c|c|c|}
\hline \multirow[b]{2}{*}{ Type of PA } & \multicolumn{2}{|c|}{ Hemorrhage } & \multirow[b]{2}{*}{$p$ Value } & \multirow[b]{2}{*}{ Sensitivity } & \multirow[b]{2}{*}{ Specificity } & \multicolumn{2}{|c|}{$\mathrm{K}(95 \% \mathrm{Cl})$} \\
\hline & Yes & No & & & & Intrarater Agreement & Interrater Agreement \\
\hline \multicolumn{8}{|c|}{ Lenticulostriate } \\
\hline Yes & 9 & 22 & \multirow{2}{*}{0.02} & \multirow{2}{*}{0.50} & \multirow{2}{*}{0.79} & \multirow{2}{*}{$0.85(0.65-1.00)$} & \multirow{2}{*}{$0.93(0.79-1.00)$} \\
\hline No & 9 & 82 & & & & & \\
\hline \multicolumn{8}{|l|}{ Thalamic } \\
\hline Yes & 16 & 50 & \multirow{2}{*}{$<0.01$} & \multirow{2}{*}{0.89} & \multirow{2}{*}{0.52} & \multirow{2}{*}{$0.58(0.28-0.88)$} & \multirow{2}{*}{$0.58(0.28-0.88)$} \\
\hline No & 2 & 54 & & & & & \\
\hline \multicolumn{8}{|l|}{ Choroidal } \\
\hline Yes & 13 & 43 & \multirow{2}{*}{0.02} & \multirow{2}{*}{0.72} & \multirow{2}{*}{0.59} & \multirow{2}{*}{$0.70(0.46-0.94)$} & \multirow{2}{*}{$0.54(0.28-0.80)$} \\
\hline No & 5 & 61 & & & & & \\
\hline
\end{tabular}

$\mathrm{PA}=$ periventricular anastomosis

0.58 for both intra- and interrater agreement). Good intra- and interrater reliability was observed for ratings of the periventricular anastomosis score $\left(\kappa_{\mathrm{w}}=0.65[95 \%\right.$ CI 0.43-0.86] for intrarater agreement and 0.70 [95\% CI 0.49-0.90] for interrater agreement).

For every type of periventricular anastomosis, the prevalence of hemorrhage was significantly higher in patients with anastomosis than in those without $(\mathrm{p}=0.02$ for the lenticulostriate type, $\mathrm{p}<0.01$ for the thalamic type, and $\mathrm{p}$ $=0.02$ for the choroidal type; Table 1$)$. The estimated sensitivity and specificity for differentiating hemorrhage were 0.50 and 0.79 for the lenticulostriate type, 0.89 and 0.52 for the thalamic type, and 0.72 and 0.59 for the choroidal type, respectively.

\section{Association Between Periventricular Anastomosis and Hemorrhage}

Table 2 summarizes the baseline characteristics of patients with and without hemorrhagic presentation. Age and periventricular anastomosis score were significantly higher in patients with hemorrhagic presentation than in those without. No significant differences in other factors-sex, concurrent disorders, hypertension, diabetes mellitus, uni-

TABLE 2. Baseline characteristics of patients with and without hemorrhage

\begin{tabular}{lllr}
\hline \multirow{2}{*}{ Variable } & \multicolumn{2}{c}{ Yes $(\mathrm{n}=18)$} & \multirow{2}{*}{$\begin{array}{c}\mathrm{N} \\
\text { Value }(\mathrm{n}=104)\end{array}$} \\
\cline { 2 - 3 } Value \\
\hline Median age (IQR) & $37.5(24.5-43.25)$ & $24(8.25-41.75)$ & 0.02 \\
\hline Female (\%) & $10(55.6)$ & $64(61.5)$ & 0.80 \\
\hline Associated disorders (\%) & 0 & $7(6.7)$ & 0.59 \\
\hline Hypertension (\%) & $3(16.7)$ & $16(15.4)$ & 1.00 \\
\hline Diabetes mellitus (\%) & 0 & $3(2.9)$ & 1.00 \\
\hline Unilateral disease (\%) & $4(22.2)$ & $20(19.2)$ & 0.75 \\
\hline $\begin{array}{l}\text { Severe hemodynamic } \\
\text { compromise (\%) }\end{array}$ & $9(50.0)$ & $62(60.2)$ & 0.45 \\
\hline Median PA score (IQR) & $2(1.75-3)$ & $1(0-2)$ & $<0.01$ \\
\hline
\end{tabular}

$\mathrm{IQR}=$ interquartile range. lateral disease, and severe hemodynamic compromisewere seen between the two groups.

As shown in Fig. 2, the prevalence of hemorrhagic presentation increased along with the periventricular anastomosis score: $2.8 \%$ for Score 0, $8.8 \%$ for Score 1, $18.9 \%$ for Score 2 , and $46.7 \%$ for Score 3 ( $p<0.01$ for trend). The cutoff score determined by the receiver operating characteristic curve was 2 . The sensitivity and specificity for differentiating hemorrhagic presentation at the cutoff were 0.78 and 0.63 , respectively (area under the curve 0.77 ).

Both the age variable and periventricular anastomosis score, statistically significant variables in the univariate analysis, were incorporated into the logistic regression model. Age was significantly associated with hemorrhagic presentation (OR 1.05 [95\% CI 1.01-1.09]). Multivariate ORs of hemorrhage for the 2 highest periventricular anastomosis score groups relative to the lowest score group (Score 0 ) exceeded 1 and were statistically significant (OR for Score 2, 8.92 [95\% CI 1.40-175.04]; OR for Score 3,

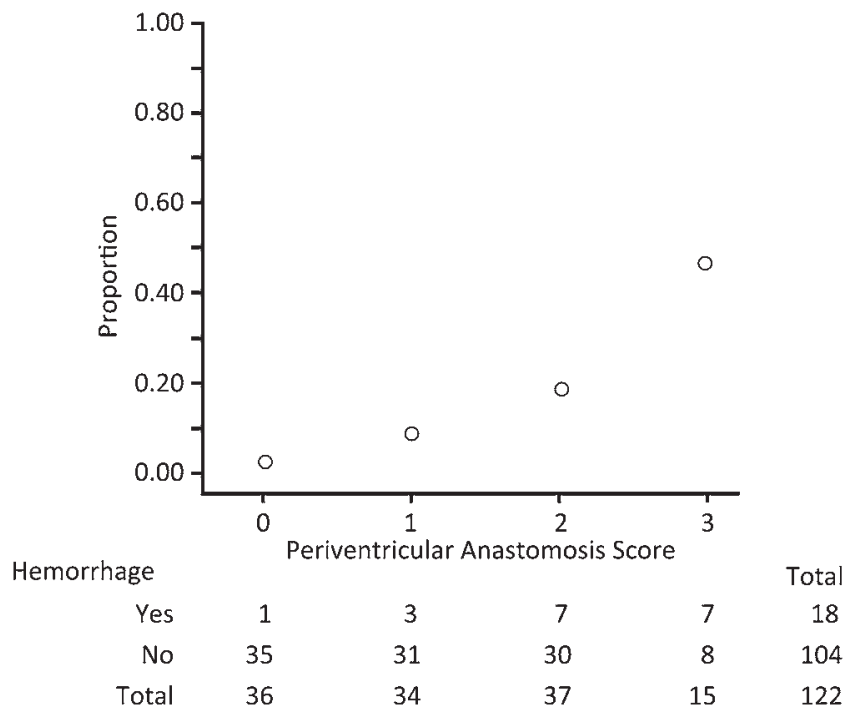

FIG. 2. Prevalence of hemorrhage relative to periventricular anastomosis score. $p<0.01$ for trend. 
TABLE 3. Multivariate odds ratio for hemorrhage according to periventricular score (adjusted for age)

\begin{tabular}{ccc}
\hline PA Score & Coefficient & Multivariate OR $(95 \% \mathrm{CI})$ \\
\hline 0 & 0 & 1.00 (reference) \\
\hline 1 & 1.18 & $3.26(0.38-68.8)$ \\
\hline 2 & 2.19 & $8.92(1.40-175.04)$ \\
\hline 3 & 3.66 & $38.84(5.46-811.48)$ \\
\hline Every 1-point increase & 1.22 & $3.38(1.84-7.00)$ \\
\hline
\end{tabular}

38.84 [95\% CI 5.46-811.48]) (Table 3 and Fig. 3). The multivariate OR for score 1 relative to score 0 also exceeds 1 , although it was not statistically significant (OR 3.26 [95\% CI 0.38-68.8]). As the relationship between periventricular anastomosis score and estimated coefficient was approximately linear, the score was incorporated with the age variable into the logistic regression model as an interval variable. Multivariate OR for every 1-point increase in the score was 3.38 (95\% CI 1.84-7.00), which was statistically significant (Table 3 ).

\section{Discussion}

Our results suggest that periventricular anastomosis is independently associated with hemorrhagic presentation in moyamoya disease. These results are in line with widely accepted speculation that abnormal fragile collaterals typical of moyamoya disease cause hemorrhage. Several examples of cerebral microbleeds suggest the presence of a certain vascular pathology in the periventricular area. The results of a meta-analysis by Qin et al. revealed that periventricular white matter was the most common site where microbleeds associated with moyamoya disease were detected. ${ }^{18}$ Kazumata et al. demonstrated that cerebral microbleeds observed in the periventricular area were more likely to be associated with hemorrhage from moyamoya disease than those in other locations. ${ }^{11}$ Sun et al. documented in the cohort study that microbleeds in the deep periventricular white matter were an independent predictor for subsequent intraventricular hemorrhage. ${ }^{20}$ Periventricular anastomosis might sufficiently explain these phenomena, given that the anastomosis might be related to hemorrhage.

A traditional theory of vascular supply in the periventricular area might be the key to understanding the development of periventricular anastomosis. Van den Bergh hypothesized 2 terminal arteries in the periventricular area, the ventriculofugal subependymal artery and ventriculopetal medullary artery. ${ }^{23,24}$ These arteries form no anastomosis in the normal brain, ${ }^{3,23}$ as the border zone between these arteries is believed to be the cause of periventricular ischemia. Long-standing cortical ischemia in moyamoya disease, however, might induce an abnormal connection between these arteries and result in periventricular anastomosis. ${ }^{14}$ This speculation might be supported by the fact that proangiogenic factors are overexpressed in the intracranial arteries and cerebrospinal fluid in patients with moyamoya disease..$^{8,21,25}$

Several mechanisms of periventricular anastomosis

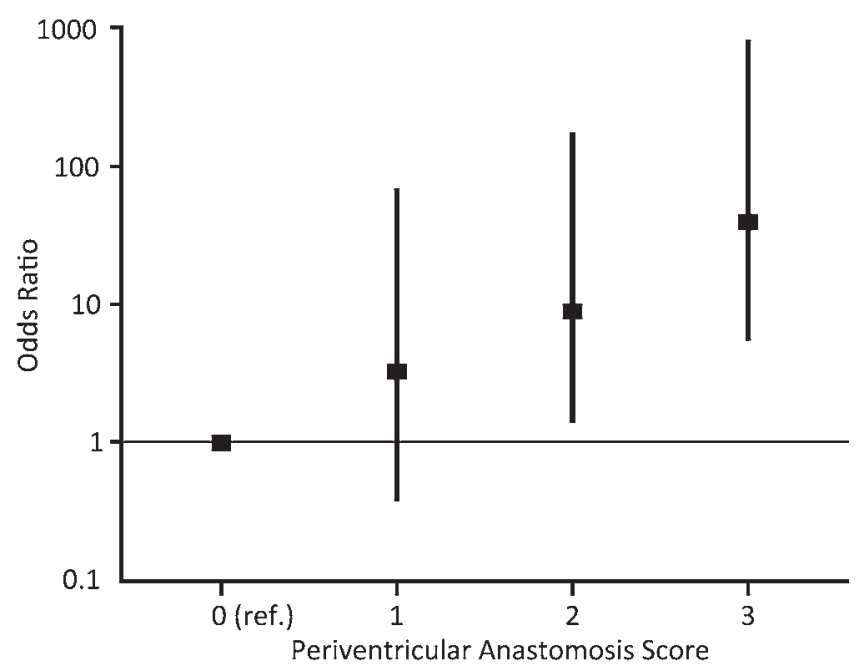

FIG. 3. Multivariate OR for hemorrhage (squares) and $95 \% \mathrm{Cl}$ (bars) according to periventricular anastomosis score. Logarithmic scale for $y$ axis. ref. $=$ reference.

causing hemorrhage can be hypothesized. First, the dilated perforating or choroidal arteries proximal to the anastomoses, serving as collaterals to the cortex, might be subject to long-standing hemodynamic stress contributing to risk of rupture. This speculation is consistent with histopathology of vascular networks in moyamoya disease, in which ruptured perforating arteries become extremely dilated. ${ }^{26}$ Second, anastomotic sites in the periventricular collateral might be especially fragile because of histologically abnormal connection between vessels. This speculation might be supported by our previous finding that microbleeds were frequently seen at the exact site of the anastomoses. ${ }^{5}$ Third, microaneurysms, another possible bleeding source in moyamoya disease, ${ }^{26}$ might grow at the site of anastomoses because of the characteristic inflection points that typically form in the collateral at these sites.

In our results, intra- and interrater reliability of the identification of periventricular anastomosis was generally acceptable, and intra- and interrater reliability of the scoring for periventricular anastomosis was good. This suggests that an MRA-based scoring system for abnormal collaterals could become a feasible scale for estimating bleeding risk. Morioka et al. proposed an angiography-based grading system for "moyamoya vessels" and collaterals from the posterior communicating and choroidal arteries. ${ }^{17}$ The reproducibility of such an angiographic grading system, however, has not been assessed. Our results are consistent with their primary finding that "dilatation and branch extension of the posterior communicating and choroidal arteries"-probably representing thalamic and choroidal periventricular anastomosis in our definition-was associated with hemorrhage. On the other hand, our finding that the lenticulostriate type was also significantly associated with hemorrhage is inconsistent with their result, in which "moyamoya vessels" were less likely to be related to hemorrhage. The subtypes of periventricular anastomosis might vary in terms of hemorrhage risk. Further studies are necessary to verify or refine scoring of periventricular anastomosis. 
Hemorrhage is a devastating manifestation of moyamoya disease, ${ }^{13}$ and risk estimates for hemorrhage might be a relevant issue. Periventricular anastomosis might serve as an indicator of hemorrhage risk. The results of a randomized controlled trial for hemorrhagic moyamoya disease suggest that direct bypass can prevent rebleeding. ${ }^{16}$ Shrinkage of abnormal collaterals through elimination of hemodynamic stress is a rationale for the effectiveness of bypass surgery. ${ }^{9,10}$ Periventricular anastomosis might also be used to evaluate the effectiveness of bypass surgery.

\section{Limitations}

As the present study incorporates a cross-sectional design, any causal relationship between the periventricular anastomosis and hemorrhage in moyamoya disease remains tentative. The score-dependent increase in the prevalence of the hemorrhagic presentation observed in this study supports the possibility of a causal relationship. However, the hypothesis that periventricular anastomosis can predict hemorrhage, introduced in the present results, should be tested in further prospective studies.

Although 3-T MRA appears effective at revealing abnormal collaterals in moyamoya disease, ${ }^{6}$ the limited resolution hinders detection of very tiny arteries. The direction of blood flow in the collateral vessels was also not taken into account. Further validation studies comparing MRA with a reference standard such as conventional angiography are required.

\section{Conclusions}

The results of the present study suggest that the periventricular anastomosis score based on MRA has good interrater reliability. The results also suggest that periventricular anastomosis is associated with hemorrhagic presentation at onset in moyamoya disease. Further prospective studies are needed to determine whether periventricular anastomosis might be useful for estimating hemorrhage risk in moyamoya disease.

\section{References}

1. Armitage P: Tests for linear trends in proportions and frequencies. Biometrics 11:375-386, 1955

2. Cho HJ, Song DB, Choi HY, Heo JH: Lenticulostriate-medullary artery anastomoses in moyamoya disease. Neurology 68:E21, 2007

3. De Reuck J: The human periventricular arterial blood supply and the anatomy of cerebral infarctions. Eur Neurol 5:321334, 1971

4. Ertl-Wagner BB, Bruening R, Blume J, Hoffmann RT, Mueller-Schunk S, Snyder B, et al: Relative value of sliding-thinslab multiplanar reformations and sliding-thin-slab maximum intensity projections as reformatting techniques in multisection CT angiography of the cervicocranial vessels. AJNR Am J Neuroradiol 27:107-113, 2006

5. Funaki T, Fushimi Y, Takahashi JC, Takagi Y, Araki Y, Yoshida $\mathrm{K}$, et al: Visualization of periventricular collaterals in moyamoya disease with flow-sensitive black-blood magnetic resonance angiography: preliminary experience. Neurol Med Chir (Tokyo) 55:204-209, 2015

6. Fushimi Y, Miki Y, Kikuta K, Okada T, Kanagaki M, Yamamoto A, et al: Comparison of 3.0- and 1.5-T three-dimen- sional time-of-flight MR angiography in moyamoya disease: preliminary experience. Radiology 239:232-237, 2006

7. Griswold MA, Jakob PM, Heidemann RM, Nittka M, Jellus V, Wang J, et al: Generalized autocalibrating partially parallel acquisitions (GRAPPA). Magn Reson Med 47:12021210, 2002

8. Hojo M, Hoshimaru M, Miyamoto S, Taki W, Nagata I, Asahi M, et al: Role of transforming growth factor-betal in the pathogenesis of moyamoya disease. J Neurosurg 89:623-629, 1998

9. Houkin K, Kamiyama H, Abe H, Takahashi A, Kuroda S: Surgical therapy for adult moyamoya disease. Can surgical revascularization prevent the recurrence of intracerebral hemorrhage? Stroke 27:1342-1346, 1996

10. Jiang H, Ni W, Xu B, Lei Y, Tian Y, Xu F, et al: Outcome in adult patients with hemorrhagic moyamoya disease after combined extracranial-intracranial bypass. J Neurosurg 121:1048-1055, 2014

11. Kazumata K, Shinbo D, Ito M, Shichinohe H, Kuroda S, Nakayama N, et al: Spatial relationship between cerebral microbleeds, moyamoya vessels, and hematoma in moyamoya disease. J Stroke Cerebrovasc Dis 23:1421-1428, 2014

12. Kim JK, Kim JH, Bae SJ, Cho KS: CT angiography for evaluation of living renal donors: comparison of four reconstruction methods. AJR Am J Roentgenol 183:471-477, 2004

13. Kobayashi E, Saeki N, Oishi H, Hirai S, Yamaura A: Longterm natural history of hemorrhagic moyamoya disease in 42 patients. J Neurosurg 93:976-980, 2000

14. Kodama N, Suzuki J: Cerebrovascular Moyamoya disease. IIIrd report-the study on the aging of the perforating branches and the possibility of collateral pathway. Neurol Med Chir (Tokyo) 14:55-67, 1974

15. Kuroda S, Houkin K: Moyamoya disease: current concepts and future perspectives. Lancet Neurol 7:1056-1066, 2008

16. Miyamoto S, Yoshimoto T, Hashimoto N, Okada Y, Tsuji I, Tominaga T, et al: Effects of extracranial-intracranial bypass for patients with hemorrhagic moyamoya disease: results of the Japan Adult Moyamoya Trial. Stroke 45:1415-1421, 2014

17. Morioka M, Hamada J, Kawano T, Todaka T, Yano S, Kai Y, et al: Angiographic dilatation and branch extension of the anterior choroidal and posterior communicating arteries are predictors of hemorrhage in adult moyamoya patients. Stroke 34:90-95, 2003

18. Qin Y, Ogawa T, Fujii S, Shinohara Y, Kitao SI, Miyoshi F, et al: High incidence of asymptomatic cerebral microbleeds in patients with hemorrhagic onset-type moyamoya disease: a phase-sensitive MRI study and meta-analysis. Acta Radiol 56:329-338, 2015

19. Research Committee on the Pathology and Treatment of Spontaneous Occlusion of the Circle of Willis: Guidelines for diagnosis and treatment of moyamoya disease (spontaneous occlusion of the circle of Willis). Neurol Med Chir (Tokyo) 52:245-266, 2012

20. Sun W, Yuan C, Liu W, Li Y, Huang Z, Zhu W, et al: Asymptomatic cerebral microbleeds in adult patients with moyamoya disease: a prospective cohort study with 2 years of follow-up. Cerebrovasc Dis 35:469-475, 2013

21. Takagi Y, Kikuta K, Nozaki K, Fujimoto M, Hayashi J, Imamura $\mathrm{H}$, et al: Expression of hypoxia-inducing factor-1 alpha and endoglin in intimal hyperplasia of the middle cerebral artery of patients with Moyamoya disease. Neurosurgery 60:338-345, 2007

22. Takahashi M: Magnification angiography in moyamoya disease: new observations on collateral vessels. Radiology 136:379-386, 1980

23. Van den Bergh R: Centrifugal elements in the vascular pattern of the deep intracerebral blood supply. Angiology 20:88-94, 1969 
24. Van den Bergh R: The ventriculofugal arteries. AJNR Am J Neuroradiol 13:413-415, 1992

25. Weinberg DG, Arnaout OM, Rahme RJ, Aoun SG, Batjer HH, Bendok BR: Moyamoya disease: a review of histopathology, biochemistry, and genetics. Neurosurg Focus 30(6):E20, 2011

26. Yamashita M, Oka K, Tanaka K: Histopathology of the brain vascular network in moyamoya disease. Stroke 14:50-58, 1983

\section{Disclosures}

The authors report no conflict of interest concerning the materials or methods used in this study or the findings specified in this paper.

\section{Author Contributions}

Conception and design: Funaki. Acquisition of data: Funaki, Morimoto. Analysis and interpretation of data: Funaki, Drafting the article: Funaki, Fushimi. Critically revising the article: Takahashi, Yoshida, Mineharu. Reviewed submitted version of manuscript: Takahashi, Yoshida, Takagi, Fushimi, Kikuchi, Mineharu, Okada, Morimoto, Miyamoto. Approved the final version of the manuscript on behalf of all authors: Funaki. Statistical analysis: Funaki. Study supervision: Takahashi.

\section{Correspondence}

Takeshi Funaki, Department of Neurosurgery, Kyoto University Graduate School of Medicine, 54 Kawahara-cho, Shogoin, Sakyo-ku, Kyoto, 606-8507, Japan. email: tfunaki@kuhp.kyoto-u. ac.jp. 